\title{
PABPC1 Enables Cells with the Suspension Cultivation Feature
}

Xiaofeng Dai ${ }^{1,2^{*} \# \text {, Yujie Miao }{ }^{3 \#} \text {, Peiyu Han }{ }^{1} \text {, Xuanhao Zhang }{ }^{3} \text {, Siming Yang }}$, Qing $\mathrm{Lv}^{4}$, Dong Hua ${ }^{4}$

${ }^{1}$ Wuxi School of Medicine, Jiangnan University, Wuxi 214122, China

${ }^{2}$ Wuhan Ammunition Life-tech Company, Ltd., Wuhan, Hubei, 430200, China

${ }^{3}$ School of Biotechnology, Jiangnan University, Wuxi 214122, China

${ }^{4}$ Affiliated Hospital of Jiangnan University, Wuxi 214000, China

* Address correspondence to:

Xiaofeng Dai:1281423490@qq.com

Wuxi School of Medicine, Jiangnan University, 1800 Lihu Avenue, Wuxi, Jiangsu 214122, China.

Wuhan Ammunition Life-tech Company, Ltd., Wuhan, Hubei, 430200, China

\# Both authors contribute equally to this work 


\section{Supplementary materials}

Supplementary Table 1. Q-PCR Primers used in this study.

\begin{tabular}{lc}
\hline \multicolumn{1}{c}{ Primer name } & Sequence \\
\hline qPCR-BHK-PABPC1-1367-F & CTGCTCCTCGACCACCATTT \\
qPCR-BHK-PABPC1-1417-R & TGTGTGGACATGACTCGTGG \\
qPCR-BHK-GAPDH-450-F & CACCACCAACTGGCTGAAATG \\
qPCR-BHK-GAPDH-524-R & GTAGTGTTGGAACCCAGTGC \\
qPCR-CHO-PABPC1-1281-F & AATGGCAAGTGTGCAAGCTG \\
qPCR-CHO-PABPC1-1386-R & AGCACGGTTCTGAGTCTGTG \\
qPCR-CHO-GAPDH-783-F & CGTGTCCGTTGTGGATCTGA \\
qPCR-CHO-GAPDH-1217-R & AGCCCCTCCTTATTCTGGGG \\
\hline
\end{tabular}

Supplementary Table 2. SiRNA sequence used in this study.

\begin{tabular}{lc}
\hline \multicolumn{1}{c}{ siRNA name } & Sequence \\
\hline$P A B P C 1$-BHK-803 & GCUAUGGGUUUGUCCACUUTT \\
$P A B P C 1$-BHK-2186 & CCGAGUCUCUCCGCUCAAATT \\
$P A B P C 1$-CHO-750 & GCAAGCCAGUACGCAUCAUTT \\
$P A B P C 1$-CHO-997 & GCUUCUAAAUGAUCGCAAATT \\
\hline
\end{tabular}

Supplementary Table 3. Summarized statistics of RNA-Seq raw reads.

\begin{tabular}{ccccccc}
\hline $\begin{array}{c}\text { Sample } \\
\text { name }\end{array}$ & $\begin{array}{c}\text { No. Initial } \\
\text { basepairs }\end{array}$ & $\begin{array}{c}\text { No. Initial } \\
\text { fragments }\end{array}$ & $\begin{array}{c}\text { No. Effective } \\
\text { fragments }\end{array}$ & $\begin{array}{c}\text { Fraction of } \\
\text { effective } \\
\text { fragments }\end{array}$ & Q30 & $\begin{array}{c}\text { GC } \\
\text { Content }\end{array}$ \\
& & & & $\mathbf{( \% )}$ & $\mathbf{( \% )}$ & $\mathbf{( \% )}$ \\
\hline BHK-adh-1 & $7.762 \mathrm{E}+09$ & 62096426 & 61285108 & 98.67 & 97.31 & 49
\end{tabular}




\begin{tabular}{ccccccc} 
BHK-adh-2 & $7.15 \mathrm{E}+09$ & 57197658 & 56231370 & 98.29 & 96.91 & 48.5 \\
BHK-adh-3 & $7.187 \mathrm{E}+09$ & 57493470 & 56679444 & 98.56 & 97.19 & 49 \\
BHK-sus-1 & $7.696 \mathrm{E}+09$ & 61571288 & 60631786 & 98.45 & 97.17 & 48 \\
BHK-sus-2 & $7.354 \mathrm{E}+09$ & 58832438 & 57966190 & 98.51 & 97.17 & 47.5 \\
BHK-sus-3 & $7.964 \mathrm{E}+09$ & 63708294 & 60925824 & 95.61 & 95.76 & 47 \\
CHO-adh-1 & $7.192 \mathrm{E}+09$ & 47948034 & 46280072 & 96.47 & 92.76 & 50 \\
CHO-adh-2 & $7.121 \mathrm{E}+09$ & 47475840 & 45144974 & 95.03 & 91.62 & 50 \\
CHO-adh-3 & $7.263 \mathrm{E}+09$ & 48422732 & 46035502 & 95.01 & 91.58 & 50 \\
CHO-sus-1 & $7.206 \mathrm{E}+09$ & 48043074 & 45850230 & 95.38 & 92.08 & 49.5 \\
CHO-sus-2 & $7.274 \mathrm{E}+09$ & 48491422 & 46887874 & 96.65 & 93.63 & 49 \\
CHO-sus-3 & $7.309 \mathrm{E}+09$ & 48725236 & 47411224 & 97.26 & 94.09 & 49 \\
\hline
\end{tabular}

\section{Supplementary Table 4. Summarized statistics of samples aligned to the reference} genome.

\begin{tabular}{cccccc}
\hline Sample name & $\begin{array}{c}\text { No. Effective } \\
\text { fragments }\end{array}$ & \multicolumn{2}{c}{$\begin{array}{c}\text { Fragments mapped to } \\
\text { the reference genome }\end{array}$} & \multicolumn{2}{c}{ Unique mapping } \\
& & No. & \% & No. & $\%$ \\
\hline BHK-adh-1 & 61285108 & 52166054 & 85.12 & 50505050 & 82.41 \\
BHK-adh-2 & 56231370 & 47879812 & 85.15 & 46402325 & 82.52 \\
BHK-adh-3 & 56679444 & 48192798 & 85.03 & 46729003 & 82.44 \\
BHK-sus-1 & 60631786 & 50816617 & 83.81 & 49433376 & 81.53 \\
BHK-sus-2 & 57966190 & 48547440 & 83.75 & 47229991 & 81.48 \\
BHK-sus-3 & 60925824 & 50775963 & 83.34 & 49410794 & 81.1 \\
CHO-adh-1 & 46280072 & 41748809 & 90.21 & 40604872 & 87.74 \\
CHO-adh-2 & 45144974 & 40962482 & 90.74 & 39846755 & 88.26 \\
CHO-adh-3 & 46035502 & 41706873 & 90.6 & 40590301 & 88.17
\end{tabular}




\begin{tabular}{llllll} 
CHO-sus-1 & 45850230 & 41593663 & 90.72 & 40497837 & 88.33 \\
CHO-sus-2 & 46887874 & 43643786 & 93.08 & 42551304 & 90.75 \\
CHO-sus-3 & 47411224 & 43649331 & 92.07 & 42542896 & 89.73 \\
\hline
\end{tabular}

